\title{
In-situ measurement and dynamic compensation of thermocouple time constant in nuclear reactors
}

\author{
V.Arunprasath ${ }^{1 *}$, Jigar V Patel $^{2}$ and Kallol Roy ${ }^{2}$ \\ Research Reactor Design and Projects Division, Bhabha Atomic Research Centre, Mumbai, India ${ }^{1}$ \\ Research Reactor Maintenance Division, Bhabha Atomic Research Centre, Mumbai, India ${ }^{2}$
}

\section{(C2016 ACCENTS}

\begin{abstract}
Periodic measurement of response time of thermocouples is essential to ensure the safe operation of a nuclear reactor. However, in order to avoid human access to radiation areas, it is required to be done in-situ. In this paper, the technique of two thermocouple probe to compute time constant is discussed and a system identification approach for the same is presented. Bandwidth of a thermocouple is limited by its physical properties and fluid properties. Also, robust thermocouple assemblies used in harsh environment usually have large time constants, which may not meet the requirement of safe, fast and efficient temperature measurement. In this paper, Kalman filter is utilized to estimate and hence reconstruct the input temperature to a thermocouple with dynamic compensation. With a known model of the thermocouple, cast in an appropriate form, the filter parameters are tuned to achieve desired response time.
\end{abstract}

\section{Keywords}

System modelling \& identification, Estimation and fault detection, Process control and instrumentation, Process automation.

\section{Introduction}

Thermocouples are one of the most widely used temperature sensors in nuclear reactors. The dynamic response characteristics of thermocouples used in critical systems play an important role in safe and efficient reactor operation. As per regulatory requirements, it is necessary to measure response time of such thermocouples periodically, in order to ensure that there is no ageing related degradation [1, 2].Conventional response time measurement techniques involve removal of a thermocouple from its mounted position and testing it in laboratory with simulated plant conditions.

However, this does not take into account the noise in the process environment, installation effects, etc. and hence the actual in-service response time is not obtained. This procedure of sensor removal from the field location may also require plant shutdown and increase the human exposure to radiation. Considering the above, in-situ response time measurement techniques were developed.

\footnotetext{
*Author for correspondence
}

The in-situ response time measurement methods are electrical heating methods, mainly the loop current step response (LCSR) test, which is a non-parametric curve fitting technique. However, such a Joule method, while resulting in fairly accurate timeconstant computation in RTDs, does not apply totally for a thermocouples, owing to the difference in the fundamental temperature sensing principle of a thermocouple (Seebeck effect) and corresponding application of either Peltier effect or Joule effect for electrical heating.

Hence it is proposed to use a System Identification approach for obtaining a parametric estimation of the model parameters of a thermocouple, which results in an indirect computation of its time constant. In this paper, the technique of two thermocouple probe is discussed and a system identification approach for the same is presented.

The bandwidth of the thermocouple is limited by its physical parameters like tip diameter, wire length etc., and is dependent on the fluid properties like Reynolds number, Nusselt number etc.

Also, robust thermocouple assemblies having protective sheaths and thermowells make them respond slowly. This may not meet the requirement 
of safe, fast and efficient temperature measurement as per technical specifications of a plant like nuclear reactor [3]. So, any effort to reduce the time constants of these thermocouples will ensure plant safety in case of abnormal temperature excursions.

Dynamic compensation is the improvement of the thermocouple's frequency response characteristics, by means of effective algorithms without any additional hardware. If the sensor dynamics are known, they can be compensated by reconstructing the sensor input from the sensor output by means of an inverse model. But the differentiating behaviour of the inverse model amplifies noise in real time signals. Hence, special methods have to be used to reduce the noise amplification. State estimation techniques like Kalman filter which make use of the known sensor model and sensor measurements can be applied for better compensation because of their tunable nature $[10,11]$. In this paper, Kalman filter is utilized to estimate and hence reconstruct the input temperature to a thermocouple. With a known model of the thermocouple, cast in an appropriate form, the filter parameters are tuned to achieve desired response time.

\section{Time constant measurement}

Time constant of a sensor is an indication of how fast a sensor is responding to a change in the process parameter. The time constant of a first order sensor is defined as the time it takes for its step response to reach $1 /$ e (i.e. $63.2 \%$ ) of its final value. It can be determined from the 'Newton's law of cooling' and is given by,

$\tau=\frac{\rho c d^{2}}{4 N_{u} k}$

where $\rho$ is the density of the sensor, $\mathrm{d}$ is the tip diameter, $\mathrm{c}$ is the specific heat of sensor material, $\mathrm{Nu}$ is the Nusselt number, and $\mathrm{k}$ is the thermal conductivity. Calculation of time constant involves knowledge of fluid properties and the physical dimensions of the sensor.

Experimentally, time constant is determined by plunge test, in which the thermocouple is given a step change by suddenly immersing the sensor in a tank of stirred water or water flowing at $1 \mathrm{~m} / \mathrm{s}$.

In-situ measurement of time constant involves Joule's heating techniques, in which the sensor is heated with an electric current and the resultant transients are analysed to compute the time constant $[3,5]$. The LCSR Test is the widely used Joule's 126 heating technique. This method has problems like requirement of high current and resulting damage to the sensor on repeated heating [4], irregularly shaped transients involving overshoots [9], errors due to magnetic effect on Type $\mathrm{K}$ thermocouples and poor accuracy [4]. Most importantly, the heating of whole body of thermocouple wires makes it cool down slowly and gives large and highly erroneous response times.

\section{Two thermocouple probe technique}

Owing to the inadequacy of the conventional methods, a novel method to compute time constant was developed based on temperature signals from two thermocouples [6].

This method called a two thermocouple probe, consists of two thermocouples of unequal diameters installed in the same process with their tips close to each other.

The first order equations of the two thermocouples are given by,

$T_{g 1}(t)=T_{1}(t)+\tau_{1} d T_{1}(t) / d t$
$T_{g 2}(t)=T_{2}(t)+\tau_{2} d T_{2}(t) / d t$

where $\tau_{1}$ and $\tau_{2}$ are the time constants, $T_{1}$ and $T_{2}$ are the sensor temperatures and, $T_{g 1}$ and $T_{g 2}$ are the input temperatures of the thermocouples 1 and 2 respectively.

Time constants of the sensors are found out by solving equations (4) and (5) assuming that their input temperatures are equal i.e. $T_{g 1}=T_{g 2}=T_{g}$. This method relies upon an a priori knowledge of the time-constant ratio defined by $\alpha=\frac{\tau_{1}}{\tau_{2}}$, which is assumed invariant [6].

Hung et al., [7] solved the equations in discrete domain making a similar assumption $T_{g 1}(k)=$ $T_{g 2}(k)=T_{g}(k)$ but without using $\alpha$. Tagawa et al., [8] solved the problem using a least squares method by finding out the time constants which minimizes the sum of squares of error between $T_{\mathrm{g} 1}$ and $\mathrm{T}_{\mathrm{g} 2}$.

Most of the existing methods solve the problem by assuming equal input temperatures and computing the value of time constants which minimises the difference between $\mathrm{T}_{\mathrm{g} 1}$ and $\mathrm{T}_{\mathrm{g} 2}$.

However, these methods fail to give good results when $\alpha$ varies or when $\mathrm{T}_{\mathrm{g} 1}(\mathrm{k}) \neq \mathrm{T}_{\mathrm{g} 2}(\mathrm{k})$. 


\section{System identification applied to a two thermocouple technique}

The equations (2) and (3) presented in section 3 are discretized at a sampling interval $\mathrm{T}_{\mathrm{s}}$ with a zero order hold as:

$$
\begin{aligned}
& T_{1}(k)=a_{1} T_{1}(k-1)+\left(1-a_{1}\right) T_{g 1}(k-1) \\
& T_{2}(k)=a_{2} T_{2}(k-1)+\left(1-a_{2}\right) T_{g 2}(k-1)
\end{aligned}
$$

where,

$a_{1}=\exp \left(\frac{-T_{S}}{\tau_{1}}\right) ; a_{2}=\exp \left(\frac{-T_{S}}{\tau_{2}}\right)$

Since $\mathrm{T}_{\mathrm{g} 1}$ and $\mathrm{T}_{\mathrm{g} 2}$ may not follow the ideal condition $\mathrm{T}_{\mathrm{g} 1}=\mathrm{T}_{\mathrm{g} 2}$, let us assume,

$T_{g 2}=T_{g 1}+e$

Where e denotes the relative offset error between the two input temperatures which may occur if they are not mounted close enough. The error e also accounts for any offset introduced by the measurements i.e. by the sensors.

$$
\begin{aligned}
& \text { Equation (5) becomes, } \\
& \begin{aligned}
\mathrm{T}_{2}(\mathrm{k})= & \mathrm{a}_{2} \mathrm{~T}_{2}(\mathrm{k}-1)+\left(1-\mathrm{a}_{2}\right) \mathrm{T}_{\mathrm{g} 1}(\mathrm{k}-1)+ \\
& \left(1-\mathrm{a}_{2}\right) \mathrm{e}
\end{aligned}
\end{aligned}
$$

The unknown temperature $\mathrm{T}_{\mathrm{g} 1}(\mathrm{k}-1)$ is eliminated from (7) and (9) to yield the following relationship between the thermocouple outputs:

$$
\begin{aligned}
\mathrm{T}_{2}(\mathrm{k})= & \mathrm{a}_{2} \mathrm{~T}_{2}(\mathrm{k}-1)+\frac{\left(1-\mathrm{a}_{2}\right)}{\left(1-\mathrm{a}_{1}\right)} \mathrm{T}_{1}(\mathrm{k})- \\
& \mathrm{a}_{1} \frac{\left(1-\mathrm{a}_{2}\right)}{\left(1-\mathrm{a}_{1}\right)} \mathrm{T}_{1}+\left(1-\mathrm{a}_{2}\right) \mathrm{e}
\end{aligned}
$$

The offset error term can be treated as a constant G. Equation (9) is non linear in parameters a1 and a2. Hence it can be represented as a 4 parameter equation as follows.

$\mathrm{T}_{2}(\mathrm{k})=$

$\theta_{1} \mathrm{~T}_{2}(\mathrm{k}-1)+\theta_{2} \mathrm{~T}_{1}(\mathrm{k})+\theta_{3} \mathrm{~T}_{1}(\mathrm{k}-$

1) $+\mathrm{G}$

By choosing $\mathrm{T}_{2}(\mathrm{k})$ as the output variable and $\mathrm{T}_{1}(\mathrm{k})$ as input variable, (10) forms an ARX structure with parameters $\theta_{1}, \theta_{2}, \theta_{3}$ and $G$. Natural random fluctuations in $\mathrm{T}_{1}(\mathrm{k})$ and $\mathrm{T}_{2}(\mathrm{k})$ form the input and output in equation (10). The regression equation is $Y=X \theta+G 1_{n}$
$Y=\left[\begin{array}{ll}X & 1_{n}\end{array}\right]\left[\begin{array}{l}\theta \\ G\end{array}\right]$

where $1_{n}$ is an $n X 1$ vector of $1 \mathrm{~s}$. Identification technique using Least squares can be applied to estimate the parameters of equation (12) by minimizing the mean-square prediction error over $\mathrm{N}$ samples, given by

$J(\theta, G)=\sum_{k=1}^{N}\left(T_{2}(k)-\widehat{T}_{2}(k)\right)^{2}$

The parameters identified can be used to find $a_{1}$ and $a_{2}$. Then, from (6), the time constants of the thermocouples can be computed by,

$$
\tau_{1}=\frac{T_{s}}{\ln \frac{1}{a_{1}}} ; \quad \tau_{2}=\frac{T_{S}}{\ln \frac{1}{a_{2}}}
$$

\section{Improvement of response time using kalman filter}

Given a change in the process temperature $\mathrm{T}_{\mathrm{g}}(\mathrm{k})$, the thermocouple output $\mathrm{T}(\mathrm{k})$ follows it with a time lag $\tau$, which is the time constant of the thermocouple. The virtual response time of the thermocouple can be reduced by reconstructing the input $\mathrm{T}_{\mathrm{g}}(\mathrm{k})$ by state estimation which forced the thermocouple response $\mathrm{T}(\mathrm{k})$.

The model of the thermocouple is assumed to be known accurately and is given by

$\mathrm{T}(\mathrm{k})=\mathrm{aT}(\mathrm{k}-1)+(1-\mathrm{a}) \mathrm{T}_{\mathrm{g}}(\mathrm{k}-1)$

whereas the model of the input, which is to be reconstructed is unknown and is assumed to be $\mathrm{T}_{\mathrm{g}}(\mathrm{k})=\mathrm{T}_{\mathrm{g}}(\mathrm{k}-1)$,

because no better model for the unknown input is available. Equation (16) is very inaccurate and hence, a large process noise covariance $\sigma_{2}^{2}$ will be assumed for this state in the model to allow changes in $\mathrm{T}_{\mathrm{g}}$.

Equations (15) and (16) are combined to get the state space model of the thermocouple as

$$
\begin{aligned}
& {\left[\begin{array}{c}
\mathrm{T}(\mathrm{k}) \\
\mathrm{T}_{\mathrm{g}}(\mathrm{k})
\end{array}\right]=\left[\begin{array}{cc}
\mathrm{a} & 1-\mathrm{a} \\
0 & 1
\end{array}\right]\left[\begin{array}{c}
\mathrm{T}(\mathrm{k}-1) \\
\mathrm{T}_{\mathrm{g}}(\mathrm{k}-1)
\end{array}\right]+\mathrm{w}(\mathrm{k}-1)} \\
& \mathrm{T}(\mathrm{k})=\left[\begin{array}{ll}
1 & 0
\end{array}\right]\left[\begin{array}{c}
\mathrm{T}(\mathrm{k}) \\
\mathrm{T}_{\mathrm{g}}(\mathrm{k})
\end{array}\right]+\mathrm{v}(\mathrm{k})
\end{aligned}
$$

The unknown input is transformed to an additional state to be estimated.

$\mathrm{w}(\mathrm{k})$ and $\mathrm{v}(\mathrm{k})$ are the process and measurement noise respectively, assumed to be zero mean, 
independent (of each other), white, and with normal probability distributions,

$\mathrm{p}(\mathrm{w}) \sim \mathrm{N}(0, \mathrm{Q}) \quad ; \mathrm{p}(\mathrm{v}) \sim \mathrm{N}(0, \mathrm{R})$

The covariance matrices $\mathrm{Q}$ and $\mathrm{R}$ of process and measurement noise, respectively, are

$\mathrm{Q}=\left[\begin{array}{cc}\sigma_{1}^{2} & 0 \\ 0 & \sigma_{2}^{2}\end{array}\right] \quad ; \quad \mathrm{R}=\sigma_{\mathrm{s}}^{2}$

where $\sigma_{\mathrm{s}}^{2}$ is the variance of the sensor noise which can be estimated from the measurements $\mathrm{T}(\mathrm{k}) . \sigma_{1}^{2}$ is the uncertainty in the process model i.e. the state equation of $\mathrm{T}(\mathrm{k}) \cdot \sigma_{2}^{2}$ is the variance of an assumed noise on the sensor input $\mathrm{T}_{\mathrm{g}}(\mathrm{k})$ which allows the input to vary more or less quickly depending on the magnitude of $\sigma_{2}^{2}$.

The estimated states are

$\hat{\mathrm{x}}(\mathrm{k})=\left[\widehat{\mathrm{T}}(\mathrm{k}) \widehat{\mathrm{T}}_{\mathrm{g}}(\mathrm{k})\right]^{\mathrm{T}}$

where $\widehat{\mathrm{T}_{\mathrm{g}}}(\mathrm{k})$ is the reconstructed input temperature. By choosing appropriate filter parameters the reconstructed input temperature $\widehat{T}_{\mathrm{g}}(\mathrm{k})$ is made to closely follow the original input $\mathrm{T}_{\mathrm{g}}(\mathrm{k})$.

\section{Choice of filter parameters}

Since an accurate model of the thermocouple is assumed, $\sigma_{1}^{2}$ is chosen as a small value when compared to $\sigma_{2}^{2}$. The sensor noise covariance $\sigma_{s}^{2}$ decides the confidence level on the accuracy of measurement. If the measurements are accurate i.e. if $\sigma_{s}^{2}$ is small, then the filter trusts the measurement and convergence of the estimate is fast and hence reconstruction is better.

$\sigma_{2}^{2}$ is the uncertainty in the model $T_{g}(k)=T_{g}(k-$ 1 ), which is highly inaccurate because there can be a change in the process temperature. Hence $\sigma_{2}^{2}$ should be chosen as a high value. But $\sigma_{2}^{2}$ has to be chosen carefully because, a trade-off is present. A high value enables the filter to follow fast changes of the sensor input whereas the noise on the sensor output is amplified strongly. The value of $\sigma_{2}^{2}$ required is also dependent on the value of $\sigma_{s}^{2}$. If the measurement is very reliable i.e. $\sigma_{s}^{2}$ is very small, then $\sigma_{2}^{2}$ required will be smaller than what is required for a higher $\sigma_{s}^{2}$.

\section{Simulations and results}

7.1Time constant computation

Simulation of the system identification method using a two thermocouple probe was done by modelling the two thermocouples as first order systems with different time constants. Zero mean Gaussian random noise signals are added to simulate the effect of noise. The accuracy of time constant estimates given by the error,

$$
e=100 *\left(\frac{\tau-\hat{\tau}}{\tau}\right)
$$

varied from $0.1 \%$ to $1 \%$ depending on the noise level. $\tau$ is the reference time constant (plunge test) and $\hat{\tau}$ is the calculated time constant. These results are obtained after simulations with various sensor models with different time constants Figure 1 shows the performance of the basic least squares method and system identification method to compute time constant using a two thermocouple probe with offset error. The system identification algorithm which compensates for the offset between $T_{g 1}$ and $T_{g 2}$ gives accurate results even in the presence of offset error whereas the basic least squares method becomes more erroneous with offset.

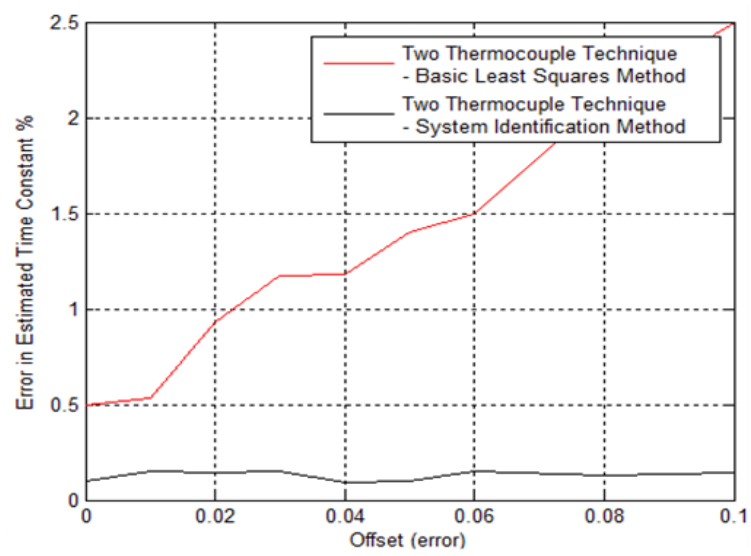

Figure 1 Performance of basic least squares method and system identification method with offset error

\subsection{Improvement of response time}

Simulations of the Kalman filter to improve the response time were done by modeling the thermocouple as a simple first order system with a time constant of 0.5 second. The algorithm is tested by giving the system a step input as shown in the Figures $2 \& 3$ and studying the improvement in response time for various values of $\mathrm{Q}$ and $\mathrm{R}$ matrix elements. It can be seen from the figures that optimum choice of $\mathrm{Q}$ and $\mathrm{R}$ matrix elements lead to a better as well as faster reconstruction. Noise in the measurement decides $\mathrm{R}$ and this has a direct effect on the quality of reconstruction and the improved response time. Hence, simulations were done with noise added to the measurements. The noise added was of Gaussian distribution, having zero mean and a 
standard deviation of $0.01 . \sigma_{1}^{2}$ is chosen as 1 while $\sigma_{2}^{2}$ and $\sigma_{s}^{2}$ are tuned to achieve the desired response of the thermocouple.

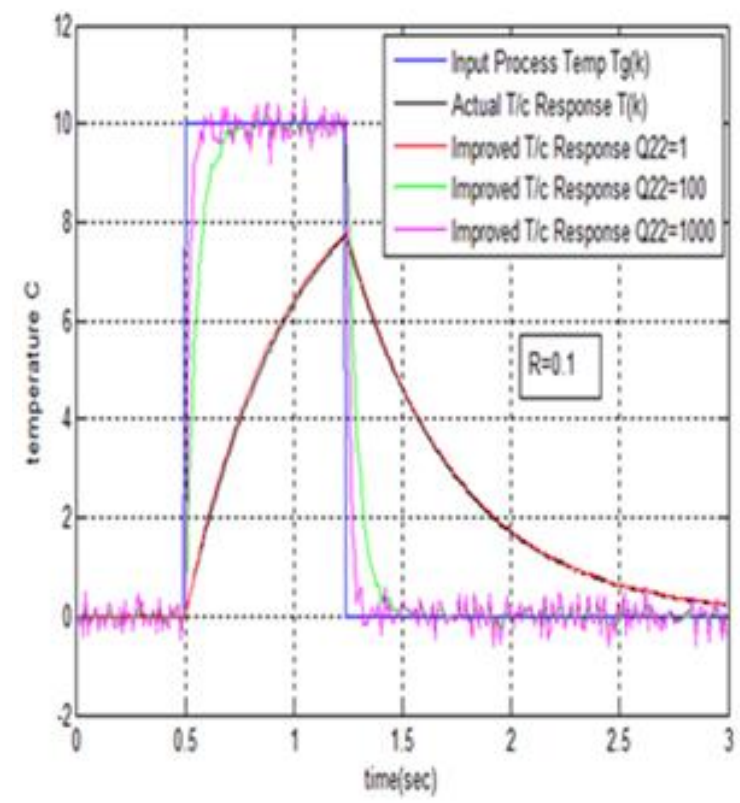

Figure 2 Actual $(\tau=0.5)$ and improved responses for various $\sigma_{2}^{2}$ with $\mathrm{R}=0.1$.

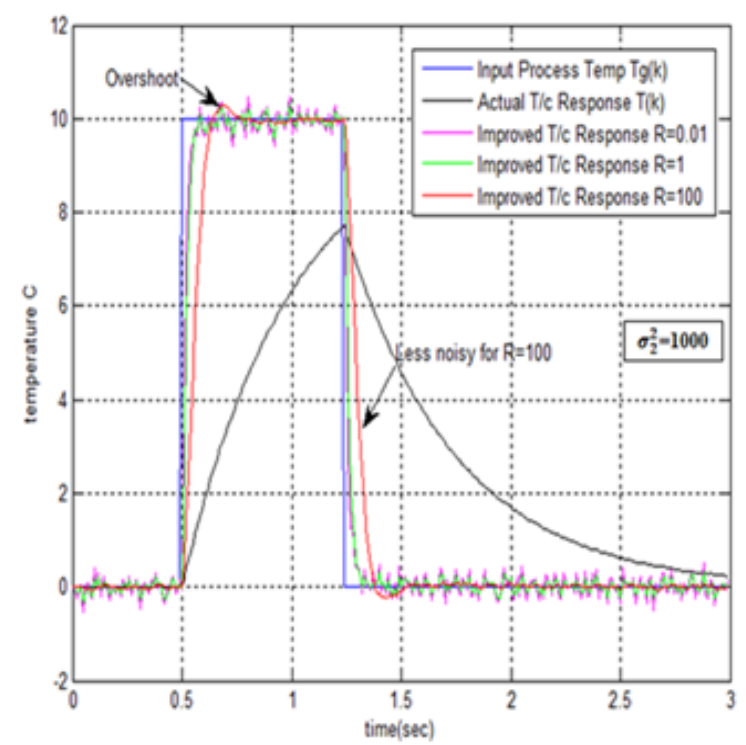

Figure 3 Actual $(\tau=0.5)$ and improved responses for various $R$ with $\sigma_{2}^{2}=1000$.

Figure 2 shows the variation in the performance of the algorithm with a fixed $\mathrm{R}$ and varying $\mathrm{Q}$. Increase in value of $\sigma_{2}^{2}$ leads to faster reconstruction. $\mathrm{Q}$ having $\sigma_{2}^{2}$ as high as 1000 provides an improved response time of 0.02 second.
At the same time, increase in $\mathrm{Q}$ also leads to a noisy reconstruction if the measurement is noisy. While increasing $\mathrm{R}$ makes the reconstruction less noisy, a large value of $R$ leads to overshoot in the reconstruction. Figure 3 shows the performance of the filter for a fixed $\sigma_{2}^{2}=1000$ and various $\mathrm{R}$ values.

It can be seen that the reconstructed temperature for $\mathrm{R}=100$ is less noisy but has an overshoot, while curves for $\mathrm{R}<100$ is corrupted with noise.

With a Kalman filter, it was observed that the factor of reduction in time constant with a good signal to noise ratio in the reconstructed signal is $10-25$. A higher factor can be achieved if the tolerable noise level is high.

\section{Conclusion}

The requirement of in-situ response time measurement and improvement of bandwidth of temperature sensors exists in applications like nuclear reactors. This paper discussed the conventional methods followed and presented the proposed techniques for the same.

The two thermocouple probe technique to compute time constants makes use of natural random fluctuations in the process temperature. It can be used as an effective alternative for the conventional current heating methods. The method presented in this paper involves modelling of the offset error between the two input temperatures and application of parameter identification to compute time constant. This avoids the assumption of a constant time constant ratio and equal input temperatures. Simulation results show that the time constant estimates agree with the standard plunge test results with less than $1 \%$ error.

Dynamic compensation of thermocouples using Kalman filter effectively reconstructs the input temperatures without model inversion and the associated noise amplification. Optimum filter parameters provide desired response times and signal to noise ratio in the reconstructed signal. The factor of reduction achieved with a good signal to noise ratio is $10-25$. A higher factor can be achieved if the tolerable noise level is high.

\section{Acknowledgment}

The authors would like to thank the management of Research Reactor Design \& Projects Division \& Research Reactor Maintenance Division, Bhabha Atomic Research Centre, and the management of 
Homi Bhabha National Institute for the opportunity to carry out this research activity and for their support.

\section{Conflicts of interest}

The authors have no conflicts of interest to declare.

\section{References}

[1] http://www-pub.iaea.org/books/iaeabooks/7790/Online-Monitoring-for-Improving-Performance-ofNuclear-Power-Plants-Part-1-Instrument-ChannelMonitoring. Accessed 20 October 2015.

[2] Bock HW, Burgis R, Eiler J, Hashemian HM, Kim $\mathrm{KH}$, Kononenko AI, et al. Management of life cycle and ageing at nuclear power plants: improved i\&c maintenance. Report prepared within the framework of the technical working group on nuclear power plant control and instrumentation. IAEA-TECDOC-1402; 2004.

[3] Hashemian HM. On-line monitoring and calibration techniques in nuclear power plants. AMS Corporation, AMS technology center. 2009; 9119.

[4] Hashemian HM, MITCHELL D. New technology for remote testing of response time of installed thermocouples. United States air force, Arnold engineering development center, report number AEDC-TR-91-26. 1992.

[5] Lockwood FC, Moneib HA. A new on-line pulsing technique for response measurements of thermocouple wires. Combustion Science and Technology.1981; 26(3-4):177-81.

[6] Cambray P. Measuring thermocouple time constants: a new method. Combustion Science and Technology.1986; 45(3-4):221-4.

[7] Hung PC, Irwin G, Kee R, McLoone S. Difference equation approach to two-thermocouple sensor characterization in constant velocity flow environments. Review of Scientific Instruments. 2005; 76(2):024902.

[8] Tagawa M, Ohta Y. Two-thermocouple probe for fluctuating temperature measurement in combustionrational estimation of mean and fluctuating time constants. Combustion and flame. 1997; 109(4):54960 .
[9] Park SJ, Ro ST. A new method for measuring time constants of a thermocouple wire in varying flow states. Experiments in Fluids.1996; 21(5):380-6.

[10] Kar K, Roberts S, Stone R, Oldfield M, French B. Instantaneous exhaust temperature measurements using thermocouple compensation techniques. SAE Technical Paper; 2004.

[11] O'Reilly PG, Kee RJ, Fleck R, McEntee PT. Twowire thermocouples: A nonlinear state estimation approach to temperature reconstruction. Review of Scientific Instruments. 2001; 72(8):3449-57.

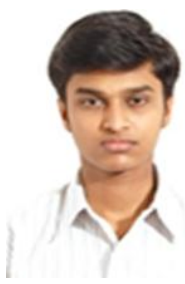

V.Arunprasath received his bachelor's degree in Electronics \& Instrumentation Engineering from SASTRA University and Master's from Homi Bhabha National Institute. Presently, he works in Research Reactor Design \& Projects Division of BARC and is from the $55^{\text {th }}$ batch of BARC training school. His current interests are Instrumentation \& Control systems of Nuclear reactors, sensor fault diagnostics and Ageing management.

Email: arunv@barc.gov.in

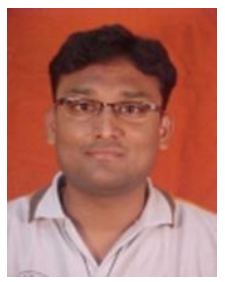

Jigar V Patel received his bachelor's degree in Inst. \& Control from DDU, Nadiad \& Master's from Homi Bhabha National Institute. Presently, he works in Research Reactor Maintenance Division of BARC and is from the $51^{\text {th }}$ batch of BARC training school. His current interests are in Ageing management, fault diagnostics and EMI modelling.

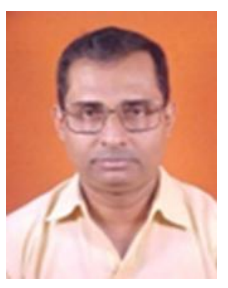

Kallol Roy is from $28^{\text {th }}$ batch of BARC training school. He received his B.Tech (Electrical) from NIT-K, M.Tech from CEDT, IISC, PhD from Syscon, IIT-B, and a Post-Doc Fellowship from University of Alberta (Canada). His primary research interests are in Fault Diagnostics, Ageing Management, Data mining, Uncertainty estimation in Instrumentation and EMI Modeling \& Computation. Presently he is with RRMD, BARC and also a faculty at HBNI. 